\title{
Derivative Process Using Fractal Indices $k$ Equals One-Half, One-Third, and One-Fourth
}

\author{
Salvador A. Loria \\ College of Engineering \& Graduate School, Nueva Ecija University of Science and Technology, Cabanatuan City, Philippines \\ Email: buddyloria2000@gamil.com
}

How to cite this paper: Loria, S.A. (2017) Derivative Process Using Fractal Indices $\mathrm{k}$ Equals One-Half, One-Third, and OneFourth. Journal of Applied Mathematics and Physics, 5, 453-461. https://doi.org/10.4236/jamp.2017.52040

Received: January 12, 2017

Accepted: February 18, 2017

Published: February 21, 2017

Copyright (C) 2017 by author and Scientific Research Publishing Inc. This work is licensed under the Creative Commons Attribution International License (CC BY 4.0).

http://creativecommons.org/licenses/by/4.0/

\begin{abstract}
In this paper, the researcher explored and analyzed the function between integral indices of derivative. It is proved that getting half-derivative twice is equivalent to first derivative. Also getting the triple of one-third derivative is equal to first derivative. Similarly, getting four times of one-fourth derivative is equal to first derivative.
\end{abstract}

\section{Keywords}

Fractal Index, Derivative, Gamma Function, Factorial

\section{Introduction}

Fractal is a general term used to express both the geometry and the procedures which display self-similarity, scale invariance, and fractional dimension [1]. Geometry deals with shapes or objects described in integral dimension. A point has 0-dimension, a line having 1-dimension, a surface has 2-dimension and the solid has 3-dimensions [2]. However, there are phenomena that are suitably characterized their dimension between any two integral dimensions. A straight line has dimension of 1 and a zigzag has dimension $n=\{d /$ s.t. $d \in(1,2)\}$. Here, the dimension is indicated as fractional dimension-a dimension whose value lies between integral values. Similarly, derivative process is an integral index in nature, such as first, second, third and up to $n^{\text {th }}$ derivative. The function obtained from derivative process is very useful in the field of physical science and technology. Thus, it is interesting to describe and analyze the function between integral indices of derivatives. Specifically, the study aimed to explore derivative process using fractal indices $\mathrm{k}$ that equals one-half, one-third, and one-fourth.

\section{The Derivative Process}

The derivative of $y$ with respect to $x$ is itself a function of $x$, and may in 
turn be differentiated [3]. The derivative of the first derivative is called second derivative and is written $\frac{\mathrm{d}^{2} y}{\mathrm{~d} x^{2}}$ or $y^{\prime \prime}$; the derivative of the second called third derivative, $\frac{\mathrm{d}^{3} y}{\mathrm{~d} x^{3}}$ or $y^{\prime \prime \prime}$, and so on...

Let $y=x^{n}$, then the derivatives are as follows::

$\frac{\mathrm{d} y}{\mathrm{~d} x}=n x^{n-1} \quad$ first derivative

$\frac{\mathrm{d}^{2} y}{\mathrm{~d} x^{2}}=n(n-1) x^{n-2} \quad$ second derivative

$\frac{\mathrm{d}^{3} y}{\mathrm{~d} x^{3}}=n(n-1)(n-2) x^{n-3} \quad$ third derivative

$\frac{\mathrm{d}^{4} y}{\mathrm{~d} x^{4}}=n(n-1)(n-2)(n-3) x^{n-4} \quad$ fourth derivative

$\frac{\mathrm{d}^{5} y}{\mathrm{~d} x^{5}}=n(n-1)(n-2)(n-3)(n-4) x^{n-5} \quad$ fifth derivative

$\cdots$

$\cdots$

Repeating the process up to $k$ times, we have

$$
\frac{\mathrm{d}^{k} y}{\mathrm{~d} x^{k}}=n(n-1)(n-2)(n-3)(n-k+1) x^{n-k} k \text { th derivative }
$$

\section{Derivatives Using Fractal Indices}

Let us consider the function between 0 and first derivative or between first and second derivative. The index fraction indicates that the derivative process is called fractal. These can be denoted as follows:

$\frac{\mathrm{d}^{1 / 2} y}{\mathrm{~d} x^{1 / 2}}$ or $y^{1 / 2^{\prime}}$ one-half derivative (between zero derivative and first derivative)

$\frac{\mathrm{d}^{3 / 2} y}{\mathrm{~d} x^{3 / 2}}$ or $y^{3 / 2^{\prime}}$ three-halves derivative (between first derivative and second derivative)

and so on

Let $y=x^{n}$ be the function, then

For $k$ th derivative, where $k$ is an element of $\frac{1}{a}$ s. t. $a \in N$, using Equation (1).

$$
\frac{\mathrm{d}^{k}}{\mathrm{~d} x^{k}}\left(x^{n}\right)=n(n-1)(n-2) \cdots \cdots \cdot(n-k+1)=\frac{n !}{(n-k) !} x^{n-k}
$$

Factorial is equivalent to gamma function [4] as

$$
n !=n \cdot \Gamma(n)=\Gamma(n+1)
$$

Thus, in gamma function

$$
\frac{\mathrm{d}^{k}}{\mathrm{~d} x^{k}}\left(x^{n}\right)=\frac{\Gamma(n+1)}{\Gamma(n-k+1)} x^{n-k} \text { for } n>0
$$


For negative integer power, $y=x^{-n}$, the derivative are as follows:

Let $y=x^{-n}$ be the function, then

$\frac{\mathrm{d} y}{\mathrm{~d} x}=(-n) x^{-n-1}$

first derivative

$\frac{\mathrm{d}^{2} y}{\mathrm{~d} x^{2}}=-n(-n-1) x^{-n-2}$ second derivative

$\frac{\mathrm{d}^{3} y}{\mathrm{~d} x^{3}}=-n(-n-1)(-n-2) x^{-n-3}$ third derivative

$\frac{\mathrm{d}^{4} y}{\mathrm{~d} x^{4}}=-n(-n-1)(-n-2)(-n-3) x^{-n-4}$ fourth derivative

$\frac{\mathrm{d}^{5} y}{\mathrm{~d} x^{5}}=-n(-n-1)(-n-2)(-n-3)(-n-4) x^{-n-5}$ fifth derivative

$\cdots$

$\cdots$

Repeating the process to $\mathrm{k}$ times, we have

$\frac{\mathrm{d}^{k} y}{\mathrm{~d} x^{k}}=-n(-n-1)(-n-2)(-n-3)(-n-k+1) x^{-n-k}$ $k$ th derivative or $\frac{\mathrm{d}^{k} y}{\mathrm{~d} x^{k}}=(-1)^{k} n(n+1)(n+2)(n+3)(n+k-1) x^{-(n+k)} \quad k$ th derivative In factorial form:

$$
\frac{\mathrm{d}^{k} y}{\mathrm{~d} x^{k}}=(-1)^{k} \frac{(n+k-1) !}{(n-1) !} x^{-(n+k)}
$$

It is equivalent to gamma function as:

$$
\frac{\mathrm{d}^{k} y}{\mathrm{~d} x^{k}}=(-1)^{k} \frac{\Gamma(n+k)}{\Gamma(n)} x^{-(n+k)}
$$

Thus,

$$
\frac{\mathrm{d}^{k}}{\mathrm{~d} x^{k}} x^{-n}=(-1)^{k} \frac{\Gamma(n+k)}{\Gamma(n)} x^{-(n+k)} \text { for } n>0
$$

\section{Gamma Function}

Definition 1. [4] [5] Gamma function is defined as $\Gamma(n)=\int_{0}^{\infty} \mathrm{e}^{-x} x^{n-1} \mathrm{~d} x$, for $n \in Z^{+}$, then,

$$
\begin{gathered}
\Gamma(n+1)=\int_{0}^{\infty} \mathrm{e}^{-x} x^{n} \mathrm{~d} x \\
\Gamma(n+1)=-\left.x^{n} \mathrm{e}^{-x}\right|_{0} ^{\infty}+\int_{0}^{\infty} n x^{n-1} \mathrm{e}^{-x} \mathrm{~d} x \\
\Gamma(n+1)=0+n \Gamma(n)=n \Gamma(n)
\end{gathered}
$$

For $\Gamma(1 / 2)$

$$
\Gamma(1 / 2)=\int_{0}^{\infty} \mathrm{e}^{-x} x^{-1 / 2} \mathrm{~d} x
$$

Let $x=u^{2}, u=x^{\frac{1}{2}}, \mathrm{~d} x=2 u \mathrm{~d} u$, 


$$
\begin{gathered}
\mathrm{d} u=\frac{\mathrm{d} x}{2 u}=\frac{\mathrm{d} x}{2 x^{\frac{1}{2}}}=2 \mathrm{~d} u=x^{-1 / 2} \mathrm{~d} x \\
\Gamma(1 / 2)=\int_{0}^{\infty} \mathrm{e}^{-u^{2}} x^{-1 / 2} \mathrm{~d} x=\int_{0}^{\infty} \mathrm{e}^{-u^{2}} 2 \mathrm{~d} u=2 \int_{0}^{\infty} \mathrm{e}^{-u^{2}} \mathrm{~d} u
\end{gathered}
$$

we can express in terms of other variable.

Thus,

$$
\Gamma(1 / 2)=2 \int_{0}^{\infty} \mathrm{e}^{-v^{2}} \mathrm{~d} v
$$

Getting the product of the two above equations, we have

$$
\left[\Gamma\left(\frac{1}{2}\right)\right]^{2}=2 \int_{0}^{\infty} \mathrm{e}^{-u^{2}} \mathrm{~d} u 2 \int_{0}^{\infty} \mathrm{e}^{-v^{2}} \mathrm{~d} v=4 \int_{0}^{\infty} \int_{0}^{\infty} \mathrm{e}^{-\left(u^{2}+v^{2}\right)} \mathrm{d} u \mathrm{~d} v
$$

let $u=r \cos \theta$ and $v=r \sin \theta$

$$
\begin{gathered}
r^{2}=u^{2}+v^{2} \\
\mathrm{~d} u \mathrm{~d} v=r \mathrm{~d} r \mathrm{~d} \theta \\
{\left[\Gamma\left(\frac{1}{2}\right)\right]^{2}=4 \int_{0}^{\frac{\pi}{2}} \int_{0}^{\infty} \mathrm{e}^{-r^{2}} r \mathrm{~d} r \mathrm{~d} \theta} \\
=4 \times-\frac{1}{2} \int_{0}^{\pi / 2} \mathrm{~d} \theta \int_{0}^{\infty} \mathrm{e}^{-r^{2}}-2 r \mathrm{~d} r \\
=-2\left[\frac{\pi}{2}-0\right][0-1] \\
=\pi
\end{gathered}
$$

Thus, $\Gamma(1 / 2)=\sqrt{\pi}=1.77245$.

The gamma function of fraction with multiple of one-half will be obtained as follows:

$$
\begin{gathered}
\Gamma(n+1)=n !=n \Gamma(n) \\
\Gamma\left(\frac{3}{2}\right)=\left(\frac{1}{2}\right) !=\Gamma\left(\frac{1}{2}+1\right)=\frac{1}{2} \Gamma\left(\frac{1}{2}\right) \\
\Gamma\left(\frac{3}{2}\right)=\frac{1}{2} !=\frac{1}{2} \sqrt{\pi}=0.88623
\end{gathered}
$$

and $\Gamma\left(\frac{5}{2}\right)=\frac{3}{2}(0.88623)=1.32934$.

Since the solution of gamma function using integral is complex, the Burnside's approximate solution [6] [7], $x !=x^{x} \mathrm{e}^{-x} \sqrt{2 \pi x}\left[1+\frac{1}{12 x}\right]$, s. t. $\mathrm{x} \in R \square\{0\}$ can be used.

Table 1 shows the actual values of gamma function and the approximate Burnside's solution. The absolute percentage of error was computed, which it is ranges between 0.06 to 1.5 percent, so Burnside's equation found acceptable.

Using Burnside's formula, $\frac{1}{2} !=\left(\frac{1}{2}\right)^{\frac{1}{2}} \mathrm{e}^{-\frac{1}{2}} \sqrt{2 \pi\left(\frac{1}{2}\right)}\left[1+\frac{1}{12\left(\frac{1}{2}\right)}\right]=0.88687$, 
Table 1. Comparison of values of gamma function of $R^{+}$.

\begin{tabular}{cccc}
\hline$\Gamma(k)$ & Actual Value & Burnside's Formula & Absolute Percentage of Error \\
\hline$\Gamma(1 / 2)$ & 1.77245 & 1.77374 & 0.07278 \\
$\Gamma(3 / 2)$ & 0.88623 & 0.88557 & 0.07447 \\
$\Gamma(5 / 2)$ & 1.32934 & 1.32884 & 0.03761 \\
$\Gamma(7 / 2)$ & 3.32335 & 3.32263 & 0.02166 \\
$\Gamma(1 / 4)$ & 3.62561 & 3.68104 & 1.52885 \\
$\Gamma(3 / 4)$ & 1.22542 & 1.22431 & 0.09058 \\
$\Gamma(5 / 4)$ & 0.90640 & 0.90559 & 0.08936 \\
$\Gamma(13 / 4)$ & 2.54926 & 2.54863 & 0.02471 \\
$\Gamma(15 / 4)$ & 4.42299 & 4.42214 & 0.01922 \\
$\Gamma(1 / 3)$ & 2.67894 & 2.67897 & 0.00112 \\
$\Gamma(2 / 3)$ & 1.35412 & 1.35321 & 0.06720 \\
$\Gamma(4 / 3)$ & 0.89298 & 0.89222 & 0.08511 \\
$\Gamma(10 / 3)$ & 2.77816 & 2.77751 & 0.02340 \\
$\Gamma(11 / 3)$ & 4.01220 & 4.01140 & 0.01994 \\
\hline & & & \\
\hline
\end{tabular}

then, $\frac{1}{2} !=\frac{1}{2} \Gamma\left(\frac{1}{2}\right)$ or we have $\Gamma\left(\frac{1}{2}\right)=1.77374$

for $\frac{3}{2} !=\left(\frac{3}{2}\right)^{\frac{1}{2}} \mathrm{e}^{-\frac{1}{2}} \sqrt{2 \pi\left(\frac{3}{2}\right)}\left[1+\frac{1}{12\left(\frac{3}{2}\right)}\right]=1.32835$

then, $\Gamma\left(\frac{3}{2}\right)=0.88557$.

\section{Roots of Negative 1}

The roots of -1 such as square roots, cube roots, fourth-roots, etc. can be obtained using the roots of complex numbers.

Definition 2. [8] Let $z \in C$, then $z=x+y i=r(\cos \theta+i \sin \theta)=r \operatorname{cis} \theta=r \mathrm{e}^{i \theta}$, sine $\theta$ is a multiple of $2 \pi$, then the general form is $z=r e^{i(\theta+2 \pi k)}$, where $k \in Z$.

Definition 3. [8] Let $z_{0}$ be the $n$th root of complex number $Z=r \mathrm{e}^{i(\theta+2 \pi k)}$ then $z_{0}=r^{1 / n} \mathrm{e}^{i(\theta+2 \pi k) / n}$ for $k=0,1,2,3 \cdots \cdots \cdots \cdot n-1$.

The principal root is the root at $k=0$, hence the principal $n$ th-root is $z_{0}=r^{1 / n} \mathrm{e}^{i \theta / n}$. Let $z=-1,=1 \mathrm{e}^{i(\pi+2 \pi k) / n}$

Taking the square-root, we have $1 \mathrm{e}^{i \frac{(\pi+2 \pi k)}{2}}=1 \mathrm{e}^{i(\pi / 2+\pi k)}$ where $k=0$ and 1.

Thus, the roots are: $i$ and $-i$ and the principal root is $i$.

Taking the cube-roots, we have $1 e^{i \frac{(\pi+2 \pi k)}{3}}$, where $k=\{0,1,2\}$.

Thus the roots are $1 / 2+\sqrt{3} / 2 i,-1,1 / 2-\sqrt{3} / 2 i$ and the principal root is $1 / 2+\sqrt{3} / 2 i$. 
Taking the fourth-roots, we have $1 \mathrm{e}^{i \frac{(\pi+2 \pi k)}{4}}$, where $k=\{0,1,2,3\}$.

Thus the roots are

$\sqrt{2} / 2+\sqrt{2} / 2 i,-\sqrt{2} / 2+\sqrt{2} / 2 i,-\sqrt{2} / 2-\sqrt{2} / 2 i, \sqrt{2} / 2-\sqrt{2} / 2 i$, and the principal root is $\sqrt{2} / 2+\sqrt{2} / 2 i$.

\section{Main Result}

For $k=1 / 2$,

let the function $y=2 x^{3}, n=3$, suppose $k=1 / 2$ then $y^{\frac{1}{2^{\prime}}}, y^{\frac{1}{2^{n}}}$ are the first and second half-derivative.

$$
\begin{aligned}
y^{\frac{1}{2^{\prime}}} & =2 \frac{\Gamma(n+1)}{\Gamma(n-k+1)} x^{n-k}=2 \frac{\Gamma(4)}{\Gamma(4-1 / 2)} x^{3-1 / 2}=2 \frac{3 !}{\Gamma\left(\frac{7}{2}\right)} x^{\frac{5}{2}} \\
& =2 \frac{6}{\frac{15}{8} \sqrt{\pi}} x^{5 / 2}=\frac{96}{15 \sqrt{\pi}} x^{5 / 2}=3.61081 x^{5 / 2}
\end{aligned}
$$

half-derivative

Differentiate again the function, we have

$$
\begin{aligned}
y^{\frac{1}{2^{\prime \prime}}} & =\frac{96}{15 \sqrt{\pi}} \frac{\Gamma(5 / 2+1)}{\Gamma(5 / 2-1 / 2+1)} x^{\frac{5}{2}-\frac{1}{2}}=\frac{96}{15 \sqrt{\pi}} \frac{\Gamma(7 / 2)}{\Gamma(3)} x^{2} \\
& =\frac{96}{15 \sqrt{\pi}} \frac{\frac{15}{8} \sqrt{\pi}}{2} x^{2}=6 x^{2} .
\end{aligned}
$$

Thus, getting half-derivative twice is equivalent to first derivative

$$
y^{\frac{1}{2^{\prime \prime}}}=\frac{\mathrm{d}^{1 / 2}}{\mathrm{~d} x^{1 / 2}} \frac{\mathrm{d}^{1 / 2} y}{\mathrm{~d} x^{1 / 2}}=\frac{\mathrm{d} y}{\mathrm{~d} x}=\frac{\mathrm{d}\left(2 x^{3}\right)}{\mathrm{d} x}=6 x^{2}
$$

Let the function $y=2 x^{-3}, n=3$, suppose $k=1 / 2$, then $y^{1 / 2^{\prime}}, y^{1 / 2^{\prime \prime}}$ are the first and second half-derivative.

$$
\begin{aligned}
& y^{\frac{1}{2^{\prime}}}=2(-1)^{k} \frac{\Gamma(n+k)}{\Gamma(n)} x^{-(n+k)}=2(-1)^{1 / 2} \frac{\Gamma(3+1 / 2)}{\Gamma(3)} x^{-(3+1 / 2)} \\
& y^{\frac{1}{2^{\prime}}}=2 i \frac{\Gamma(7 / 2)}{\Gamma(3)} x^{-(7 / 2)}=2 i \frac{3.32335}{2} x^{-7 / 2}=3.32335 i x^{-\frac{7}{2}} .
\end{aligned}
$$

Differentiate again the function, we have

$$
\begin{aligned}
y^{\frac{1}{2^{n}}} & =3.32335 i(-1)^{k} \frac{\Gamma(n+k)}{\Gamma(n)} x^{-(n+k)} \\
& =3.32335 i(-1)^{1 / 2} \frac{\Gamma(7 / 2+1 / 2)}{\Gamma(7 / 2)} x^{-(7 / 2+1 / 2)} \\
y^{\frac{1}{2^{n}}} & =3.32335 i i \frac{\Gamma(4)}{\Gamma\left(\frac{7}{2}\right)} x^{-4}=3.32335(-1) \frac{3 !}{3.32335} x^{-4}=-6 x^{-4}
\end{aligned}
$$

This completes the proof that twice of half derivative is equivalent to first derivative.

For $k=1 / 3$, 
let $y=2 x^{3}, n=3$, suppose $k=\frac{1}{3}$, then $y^{1 / 3^{\prime}}, y^{1 / 3^{\prime \prime}}, y^{1 / 3^{\prime \prime}}$, are the first, second, and third $1 / 3$ derivatives respectively.

$$
\begin{gathered}
y^{1 / 3^{\prime}}=2 \frac{\Gamma(3+1)}{\Gamma\left(3-\frac{1}{3}+1\right)} x^{3-1 / 3}=2 \frac{\Gamma(4)}{\Gamma(11 / 3)} x^{8 / 3}=2.99089 x^{8 / 3} \\
y^{1 / 3^{\prime \prime}}=2.99089 \frac{\Gamma\left(\frac{8}{3}+1\right)}{\Gamma\left(\frac{8}{3}-\frac{1}{3}+1\right)} x^{\frac{8}{3}-\frac{1}{3}}=2.99089 \frac{\Gamma\left(\frac{11}{3}\right)}{\Gamma\left(\frac{10}{3}\right)} x^{\frac{7}{3}}=4.31942 x^{\frac{7}{3}} \\
y^{1 / 3^{\prime \prime}}=4.31942 \frac{\Gamma\left(\frac{10}{3}\right)}{\Gamma(3)} x^{\frac{7}{3}-\frac{1}{3}}=6 x^{2}
\end{gathered}
$$

Thus, triple of one-third derivative is equal to first derivative.

Let $y=2 x^{-3}, n=3$, suppose $k=\frac{1}{3}$, then $y^{1 / 3^{\prime}}, y^{1 / 3^{\prime \prime}}, y^{1 / 3^{\prime \prime}}$ are the first, second, and third $1 / 3$ derivative respectively.

$$
\begin{aligned}
y^{1 / 3^{\prime}} & =2(-1)^{1 / 3} \frac{\Gamma(n+k)}{\Gamma(n)} x^{-(n+k)}=2\left(\frac{1}{2}+\frac{\sqrt{3}}{2} i\right) \frac{\Gamma\left(3+\frac{1}{3}\right)}{\Gamma(3)} x^{-\frac{10}{3}} \\
& =(1.38908+2.40596 i) x^{-\frac{10}{3}} ; \quad \Gamma\left(\frac{10}{3}\right)=2.77816 \\
y^{1 / 3^{\prime \prime}} & =(1.38908+2.40596 i) \frac{\Gamma(11 / 3)}{\Gamma(10 / 3)} x^{-\frac{11}{3}} \\
& =(-2.00609+3.47466 i) x^{-\frac{11}{3}} \\
y^{1 / 3^{\prime \prime}} & =(-2.00609+3.47466 i)\left(\frac{1}{2}+\frac{\sqrt{3}}{2} i\right) \frac{\Gamma\left(\frac{11}{3}+\frac{1}{3}\right)}{\Gamma\left(\frac{11}{3}\right)} x^{-\frac{12}{3}} \\
& =1.49544(-4.01219+0 i) x^{-4}=-6 x^{2}
\end{aligned}
$$

This completes the proof that the triple of one-third derivative is equal to first derivative

For $k=1 / 4$

Let $y=2 x^{3}, n=3$, suppose $k=\frac{1}{4}$, then $y^{1 / 4^{\prime}}, y^{1 / 4^{\prime \prime}}, y^{1 / 4^{\prime \prime \prime}}, y^{1 / 4^{\prime \prime \prime}}$ are the first, second, third and fourth $1 / 4$ derivatives respectively.

$$
\begin{aligned}
& y^{1 / 4^{\prime}}=2 \frac{\Gamma(3+1)}{\Gamma\left(3-\frac{1}{4}+1\right)} x^{3-1 / 4}=2 \frac{\Gamma(4)}{\Gamma(15 / 4)} x^{11 / 4}=2.71310 x^{11 / 4} \\
& y^{1 / 4^{\prime \prime}}=2.71310 \frac{\Gamma(15 / 4)}{\Gamma(7 / 2)} x^{5 / 2}=3.61082 x^{5 / 2} \\
& y^{1 / 4^{\prime \prime}}=3.61082 \frac{\Gamma(7 / 2)}{\Gamma(13 / 4)} x^{9 / 4}=4.70726 x^{9 / 4}
\end{aligned}
$$




$$
y^{1 / 4^{\prime \prime \prime}}=4.70726 \frac{\Gamma(13 / 4)}{\Gamma(3)} x^{2}=6 x^{2}
$$

This completes the proof that four times of one-fourth derivative is equivalent to first derivative.

Let $y=2 x^{-3}, n=3$, suppose $k=1 / 4$, then $y^{1 / 4^{\prime}}, y^{1 / 4^{\prime \prime}}, y^{1 / 4^{\prime \prime}}, y^{1 / 4^{\prime \prime \prime}}$ are the first, second, third and fourth $1 / 4$ derivatives respectively.

$$
\begin{aligned}
y^{1 / 4^{\prime}} & =2(-1)^{k} \frac{\Gamma(n+k)}{\Gamma(n)} x^{-(n+k)}=2\left(\frac{\sqrt{2}}{2}+\frac{\sqrt{2}}{2} i\right) \frac{\Gamma\left(3+\frac{1}{4}\right)}{\Gamma(3)} x^{-\left(3+\frac{1}{4}\right)} \\
& =(1.8026+1.8026 i) x^{-13 / 4} \\
y^{1 / 4^{\prime \prime}} & =(1.8026+1.8026 i)\left(\frac{\sqrt{2}}{2}+\frac{\sqrt{2}}{2} i\right) \frac{\Gamma\left(\frac{7}{2}\right)}{\Gamma\left(\frac{13}{4}\right)} x^{-\frac{15}{4}} \\
& =(2.54926 i)(1.30365) x^{-14 / 4}=3.23334 i x^{-14 / 4} \\
y^{1 / 4^{\prime \prime}} & =3.23334 i\left(\frac{\sqrt{2}}{2}+\frac{\sqrt{2}}{2} i\right) \frac{\Gamma\left(\frac{15}{4}\right)}{\Gamma\left(\frac{7}{2}\right)} x^{-\frac{15}{4}} \\
& =(-3.12752+3.12752 i) x^{-15 / 4} \\
y^{1 / 4^{\prime \prime \prime}} & =(-3.12752+3.12752 i)\left(\frac{\sqrt{2}}{2}+\frac{\sqrt{2}}{2} i\right) \frac{\Gamma(4)}{\Gamma(15 / 4)} x^{-4} \\
& =-4.42298(1.35655) x^{-4}=-6 x^{2}
\end{aligned}
$$

This completes the proof that getting the one-fourth derivatives four times is equivalent to one whole or first derivative.

\section{Conclusion}

The study explored and analyzed the function between integral indices of derivative based on the theoretical deduction of the gamma function. The above solutions and proofs confirmed that derivatives using fractal indices exist everywhere. Derivatives contributed significantly to the field of physical science. It is very interesting to describe and analyze the behavior of functions obtained through derivative process using fractal indices. Likewise, the process being used in this paper can be extended to analyze derivatives of different transcendental functions.

\section{References}

[1] Mandelbrot, B. (1982) The Fractal Geometry of Nature. W. H. Freeman and Company, San Francisco.

[2] Borres, M.S. (2012) From Fractal Geometry to Statistical Fractal.

[3] Love, C. and Rainville, E. (1981) Differential and Integral Calculus. 6th Edition, Collier-Mcmillan International Editions, Macmillan Publishing Co. Inc., Toronto, 
Ontario.

[4] Dulay, V.N. (1996) Reviewer/Text in Advanced Engineering Mathematics. Rex Book Store, Manila, Philippines.

[5] Chen, B. (2012) Some Properties on the Function Involving Gamma Function. Applied Mathematics, 3, 587-589. https://doi.org/10.4236/am.2012.36090 http://www.scirp.org/journal/PaperInformation.aspx?PaperID=20283

[6] Lu, D. (2014) A Generated Approximation Related to Burnside's Formula. Journal of Number Theory, 136, 414-422. https://doi.org/10.1016/j.jnt.2013.10.016 http://www.sciencedirect.com/science/article/pii/S0022314X13002850

[7] Shi, X., Liu, F., and Qu, H. (2010) The Burnside Approximation of Gamma Functio. Analysis and Application, 8, 315-322. https://doi.org/10.1142/S0219530510001643

[8] Brown, J.W., Churchill, R.V. (1996) Complex Variables and Applications. 6th Edition, McGraw-Hill, Inc., New York.

\section{Scientific Research Publishing}

Submit or recommend next manuscript to SCIRP and we will provide best service for you:

Accepting pre-submission inquiries through Email, Facebook, LinkedIn, Twitter, etc. A wide selection of journals (inclusive of 9 subjects, more than 200 journals) Providing 24-hour high-quality service

User-friendly online submission system Fair and swift peer-review system Efficient typesetting and proofreading procedure Display of the result of downloads and visits, as well as the number of cited articles Maximum dissemination of your research work

Submit your manuscript at: http://papersubmission.scirp.org/ Or contact jamp@scirp.org 